\title{
miR-21 enhances the protective effect of loperamide on rat cardiomyocytes against hypoxia/reoxygenation, reactive oxygen species production and apoptosis via regulating Akap8 and Bard1 expression
}

\author{
HONG SHEN $^{1-3}$, ZHIFENG YAO ${ }^{2,3}$, WEIPENG ZHAO ${ }^{2,3}$, YAPING ZHANG ${ }^{1}$, \\ CHENLING YAO $^{1}$ and CHAOYANG TONG ${ }^{1}$ \\ ${ }^{1}$ Department of Emergency Medicine; ${ }^{2}$ Shanghai Institute of Cardiology; ${ }^{3}$ Department of Cardiology, \\ Zhongshan Hospital, Fudan University, Shanghai 200032, P.R. China
}

Received December 30, 2017; Accepted June 1, 2018

DOI: $10.3892 /$ etm.2018.7047

\begin{abstract}
Effective therapies to reduce ischemia/reperfusion and hypoxia/reoxygenation injury are currently lacking. Furthermore, the effects of loperamide and microRNA (miR)-21 on hypoxia/reoxygenation injury of cardiomyocytes have remained to be elucidated. Therefore, the present study aimed to investigate the effect of loperamide and miR-21 on cardiomyocytes during hypoxia/reoxygenation injury, and to explore the underlying molecular mechanisms. H9c2 rat cardiomyocytes were pre-treated with loperamide prior to hypoxia/reoxygenation. The viability of H9c2 cells was measured with a cell counting kit 8 and apoptosis was detected with an Annexin V-phycoerythrin/7-aminoactinomycin D apoptosis kit. Furthermore, reactive oxygen species were detected with a specific kit. Genes regulated by miR-21 were screened with an mRNA chip and confirmed using reverse-transcription quantitative polymerase chain reaction analysis. The direct targeting relationship of miR-21 with certain mRNAs was then confirmed using a Dual-Luciferase Reporter Assay system. The results indicated that the apoptotic rate and reactive oxygen species levels in rat cardiomyocytes were markedly increased after hypoxia/reoxygenation treatment. Pre-treatment with loperamide significantly protected $\mathrm{H} 9 \mathrm{c} 2$ cells against apoptosis and reactive oxygen species production after hypoxia/reoxygenation. The protection was markedly decreased by miR-21 inhibitor and enhanced by miR-21 mimics. Screening for genes associated with cardiomyocyte apoptosis revealed that the relative
\end{abstract}

Correspondence to: Dr Chaoyang Tong, Department of Emergency Medicine, Zhongshan Hospital, Fudan University, Building 17, 180 Fenlin Road, Shanghai 200032, P.R. China

E-mail: drtcyang@sina.com

Key words: loperamide, miR-21, hypoxia, reoxygenation, cardiomyocytes expression of A-kinase anchoring protein 8 (Akap8) and BRCA1 associated RING domain 1 (Bard1) was consistent with the experimental results on apoptosis and reactive oxygen species. Compared with the group treated by hypoxia/reoxygenation alone, pre-treatment with loperamide markedly decreased the expression of BRCA1-interacting protein C-terminal helicase 1, Akap8 and Bard1 after hypoxia/reoxygenation. The decrease in the expression of Akap8 and Bard1 was markedly attenuated by miR-21 inhibitor and enhanced by miR-21 mimics. miR-21 mimics directly targeted the 3'-untranslated region (UTR) of Akap8 and Bard 1 mRNA to thereby decrease their expression. In conclusion, the protection of rat cardiomyocytes against hypoxia/reoxygenation-induced apoptosis and reactive oxygen species production by loperamide was markedly enhanced by miR-21. miR-21 directly targets the 3'-UTR of Akap8 and Bard1 mRNA and enhances the inhibitory effects of loperamide on Akap8 and Bard1 expression in rat cardiomyocytes after hypoxia/reoxygenation.

\section{Introduction}

Myocardial infarction is a common presentation of coronary artery disease. Each year, $>3$ million cases of ST-elevated myocardial infarction (STEMI) and 4 million cases of non-STEMI (NSTEMI) occur worldwide $(1,2)$. Reperfusion therapies, including primary percutaneous coronary intervention and fibrinolytic therapy, promptly restore the blood flow to the ischemic myocardium and limit the infarct size. However, the return of blood flow and oxygen may result in additional cardiac damage and complications, which is referred to as ischemia/reperfusion injury, or hypoxia/reoxygenation injury. The reperfusion and reoxygenation injury increases cell apoptosis and the production of reactive oxygen species (3). Despite an improved understanding of the pathophysiology of the process and encouraging results of pre-clinical trials for multiple agents, effective therapies to reduce or prevent reperfusion and reoxygenation injury have remained elusive. Most clinical trials to prevent reperfusion injury have been 
disappointing (4-6). Therapies to limit reperfusion and reoxygenation injury require further investigation.

Loperamide is an opioid-receptor agonist that acts on $\mu$-opioid receptors in the myenteric plexus. It acts in a similar manner to morphine, decreasing the activity of the myenteric plexus, which decreases the tone of longitudinal and circular smooth muscles of the intestinal wall $(7,8)$. Loperamide is used to decrease the frequency of diarrhea in gastroenteritis, inflammatory bowel disease and short bowel syndrome (9). Morphine, another $\mu$-opioid receptor agonist, was reported to protect against myocardial ischemia/reperfusion injury in rabbits (10). However, the effect of loperamide against hypoxia/reoxygenation injury of cardiomyocytes has remained elusive.

MicroRNAs (miRNAs/miRs) are a class of short non-coding RNAs that have important regulatory roles on gene expression by sequence-specific base pairing with the 3'-untranslated region (3'-UTR) of target mRNAs, promoting mRNA degradation or inhibiting their translation (11). miR-21 has been identified to be overexpressed in numerous types of solid tumor. Altered miR-21 expression reported to be associated with the proliferation, invasion and apoptosis of malignant cells via targeting and downregulating of various tumor suppressors, including programmed cell death $4(12,13)$, phosphatase and tensin homologue (PTEN) (14), B-cell lymphoma 2 and tropomyosin $1(15,16)$. In addition, miR-21 was revealed to attenuate hepatocyte hypoxia/reoxygenation injury via inhibiting the PTEN/phosphoinositide-3 kinase (PI3K)/AKT signaling pathway (17). Rapamycin suppressed hypoxia/reoxygenation-induced islet injury by upregulating miR-21 via the PI3K/AKT signaling pathway (18). However, the role of miR-21 in hypoxia/reoxygenation injury of cardiomyocytes remains elusive.

Therefore, the present study aimed to investigate the role of loperamide and miR-21 in hypoxia/reoxygenation injury of cardiomyocytes, and to explore the underlying molecular mechanisms.

\section{Materials and methods}

Reagents. Loperamide hydrochloride (cat. no. S2480) was purchased from Selleck Chemicals (Houston, TX, USA). Dulbecco's modified Eagle's medium (DMEM) was purchased from Thermo Fisher Scientific, Inc. (Waltham, MA, USA). Trypsin (Invitrogen; Thermo Fisher Scientific, Inc.), the Annexin V-phycoerythrin (PE)/7-aminoactinomycin D (7-AAD) apoptosis assay kit (KGA1017; Keygentec Inc., Jiangsu, China) and the reactive oxygen species assay kit (S0033; Beyotime Institute of Biotechnology, Haimen, China) were used in the present study. Primers and probes, TRIzol reagent, SuperScript III Reverse Transcriptase, SYBR-Green I and diethylpyrocarbonate-treated $\mathrm{H}_{2} \mathrm{O}$ were from Invitrogen (Thermo Fisher Scientific, Inc.). RNase inhibitor was purchased from Fermentas (Thermo Fisher Scientific, Inc.). SYBR qPCR mix kit was purchased from Invitrogen (4309155; Thermo Fisher Scientific, Inc.).

Cell hypoxia/reoxygenation model. H9c2 rat cardiomyocytes (cat. no. CRL-1446; American Type Culture Collection, Manassas, VA, USA) were cultured in DMEM supplemented with glucose, $10 \%$ fetal bovine serum (GE Healthcare, Chicago, IL, USA) and $1 \%$ penicillin/streptomycin at $37^{\circ} \mathrm{C}$ in a humidified atmosphere containing air with $5 \% \mathrm{CO}_{2}$ to a cell confluence of $50 \%$. Hypoxia was induced by culturing $\mathrm{H} 9 \mathrm{c} 2$ cells in DMEM without glucose in an atmosphere of $100 \% \mathrm{~N}_{2}$ Following hypoxia treatment for $6 \mathrm{~h}$, the $\mathrm{H} 9 \mathrm{c} 2$ cells were returned to normoxic conditions (air with $5 \% \mathrm{CO}_{2}$ ) for $3 \mathrm{~h}$. Experiments were performed using cells that were to be subjected to this hypoxia and reoxygenation treatment.

Cell viability assay. $\mathrm{H} 9 \mathrm{c} 2$ rat cardiomyocytes were pre-treated with $0,10,50,100$ or $200 \mathrm{mM}$ loperamide for $24 \mathrm{~h}$ prior to hypoxia and reoxygenation treatment. H9c2 cells without loperamide or hypoxia/reoxygenation treatment served as a control. The viability of $\mathrm{H} 9 \mathrm{c} 2$ cells was measured by using a cell counting kit 8 (CCK-8) cell viability assay (C0038; Beyotime Institute of Biotechnology). CCK-8 reagent was added into each well, followed by incubation for $4 \mathrm{~h}$. The absorbance was measured utilizing a microplate reader at $490 \mathrm{~nm}$. Experiments were performed in triplicate.

Transfection of miR-21 mimics and inhibitor. Transfection was performed prior to hypoxia treatment. miR-21 mimics and miR-21 inhibitor were designed and chemically synthesized (Shanghai GenePharma Co., Ltd., Shanghai, China), with homo sapiens (hsa)-miR-21 mimics: Sense, 5'-UAGCUUAUC AGACUGAUGUUGA-3' and anti-sense, 5'-AACAUCAGU CUGAUAAGCUAUU-3'; and hsa-miR-21 inhibitor, 5'-UCA ACAUCAGUCUGAUAAGCUA-3'. Carboxyfluorescein (FAM)-labeled negative control siRNA was used as control. $\mathrm{H} 9 \mathrm{c} 2$ cells were seeded in 6 -well plates at $5 \times 10^{5}$ cells/well and cultured in antibiotic-free medium for $48 \mathrm{~h}$ to achieve a confluence of $>70 \%$ on the day of transfection. The miR-21 mimics and inhibitor $(40 \mathrm{~nm})$ were transfected into cells using Lipofectamine ${ }^{\mathrm{TM}} 2000$ reagent (Invitrogen; Thermo Fisher Scientific, Inc.) under serum-free conditions for $6 \mathrm{~h}$ prior to replacing the culture supernatant with complete medium. The efficiency of transfection was determined using a Leica fluorescence microscope (Leica Microsystems, Wetzlar, Germany) for the FAM-labeled negative control siRNA. The transfection efficiency was also determined by assessing the effective upregulation and downregulation using reverse transcription-quantitative polymerase chain reaction (RT-qPCR).

Flow cytometry. H9c2 rat cardiomyocytes were divided into 5 groups: i) No loperamide, no hypoxia/reoxygenation (Control group), ii) hypoxia/reoxygenation (H/R group), iii) cells were pre-treated with $50 \mathrm{~nm}$ loperamide for $24 \mathrm{~h}$ prior to hypoxia/reoxygenation ( $\mathrm{H} / \mathrm{R}+$ loperamide group), iv) cells were pre-treated with $50 \mathrm{~nm}$ loperamide and transfected with miR-21 inhibitor prior to hypoxia/reoxygenation (H/R+loperamide+miR-21 inhibitor group) and v) cells were pre-treated with $50 \mathrm{~nm}$ loperamide and transfected with miR-21 mimics prior to hypoxia/reoxygenation (H/R+loperamide+miR-21 mimics group). Cell apoptosis was detected with the Annexin V-PE/7-AAD apoptosis assay kit using flow cytometry. The cells from each of the 5 groups were washed with PBS twice and incubated with trypsin at $37^{\circ} \mathrm{C}$ for $1 \mathrm{~min}$. Following digestion, the cell suspension was 
Table I. Primers used for polymerase chain reaction.

\begin{tabular}{lll}
\hline Gene & Primer name & Sequence (5'-3') \\
\hline Brip1 & Q-RAT-Brip1-F & CCCGTGCCGTCATAACCATA \\
Rad51b & Q-RAT-Brip1-R & GCAAAGGTTGAGTGGTGCTG \\
& Q-RAT-Rad51b-F & TACGACCCATCTGAGTGGAGC \\
Hspa14 & Q-RAT-Rad51b-R & GGGGACTTGGCGATGAGAAT \\
& Q-RAT-Hspa14-F & TGGGCTCAGATGCAAACGAT \\
Bard1 & Q-RAT-Hspa14-R & CCGATACATCCCGCTGTTCA \\
& Q-RAT-Bard1-F & CTTGCCCGTCTGGAGAAGTT \\
Akap8 & Q-RAT-Bard1-R & GGGCATCCTGATCCAACACA \\
& Q-RAT-Akap8-F & ACTACAATGCCCAGAACACCA \\
MXD4 & Q-RAT-Akap8-R & CTTGGCAATGAGCGAGTCAGA \\
& Q-RAT-MXD4-F & GAGTACCTGGAGCGTAGGGA \\
MTHFD1 & Q-RAT-MXD4-R & GTTTAGCTCGTCGTCGAAGG \\
& Q-RAT-MTHFD1-F & GTCACGACGTCATTCCGGT \\
\hline
\end{tabular}

Akap8, A-kinase anchoring protein 8; Bard1, BRCA1-associated RING domain 1; Brip1, BRCA1 interacting protein C-terminal helicase 1; Hspa14, heat shock protein family A (Hsp70) member 14; Rad 51b, RAD51 paralog B; MXD4, MAX dimerization protein 4; MTHFD1, methylenetetrahydrofolate dehydrogenase, cyclohydrolase and formyltetrahydrofolate synthetase 1; F, forward; R, reverse.

centrifuged at $400 \mathrm{xg}$ at room temperature for $5 \mathrm{~min}$. The cell pellet was resuspended with PBS and the centrifugation and resuspension steps were repeated twice. The cells were blocked with $2 \%$ bovine serum albumin (Sigma-Aldrich; Merck KGaA, Darmstadt, Germany) for $30 \mathrm{~min}$ at room temperature. 7-AAD (5 $\mu \mathrm{l})$ and Annexin V-PE $(1 \mu \mathrm{l})$ reagents were added to $100 \mu \mathrm{l}$ cell suspension, followed by incubation at room temperature for $10 \mathrm{~min}$. Cells were centrifuged at $400 \mathrm{x} \mathrm{g}$ at room temperature for $5 \mathrm{~min}$ and re-suspended with PBS three times. Cell fluorescence was then detected by flow cytometry. Data were acquired on an LSRII flow cytometer (BD Biosciences, Franklin Lakes, NJ, USA) and analyzed with FlowJo software (FlowJo, LLC, Ashland, OR, USA). Experiments were performed in triplicates.

Detection of reactive oxygen species. $\mathrm{H} 9 \mathrm{c} 2$ rat cardiomyocytes were divided into 5 groups as mentioned above. Reactive oxygen species were detected with the reactive oxygen species assay kit using dichlorofluorescein diacetate (DCFDA), following the manufacturer's protocol. DCFDA $(10 \mu \mathrm{M})$ was added to the cell suspension $\left(10^{6}\right.$ cells $\left./ \mathrm{ml}\right)$, with subsequent incubation at $37^{\circ} \mathrm{C}$ for $20 \mathrm{~min}$. After being washed with culture medium 3 times, cells were observed using a fluorescence microscope. The integrated optical density (IOD) was calculated by multiplying the area (size) and average density of fluorescence (19). Samples were evaluated using Image-Pro Plus 7 software (Media Cybernetics Inc., Rockville, MD, USA), and 6 fields of view (magnification, $\mathrm{x} 200)$ were assessed for each sample. Three repeats were performed.

mRNA chip assay and RT-qPCR. H9c2 rat cardiomyocytes were divided into 5 groups as mentioned above. After loperamide pretreatment and the following H/R treatment, genes regulated by miR-21 were screened by using an mRNA chip assay (Affymetrix GeneChip Rat Gene 1.0 ST Array; Affymetrix; Thermo Fisher Scientific, Inc.). Genes associated with cardiomyocyte apoptosis were screened out by pathway enrichment analysis. Pathway analysis was used to identify the significant pathways of differentially expressed genes according to the Kyoto Encyclopedia of Genes and Genomes, Biocarta and Reactome. Fisher's exact test was used to identify the pathways of genes that are significantly differentially expressed. The threshold of significance was defined by the $\mathrm{P}$-value and false discovery rate, and the enrichment was calculated (20-22). The results of the mRNA chip assay were confirmed by RT-qPCR. Diethylpyrocarbonate-treated water was used when handling RNA, to reduce the risk of degradation by RNases. Total RNA was extracted from cells using TRIzol reagent according to the manufacturer's protocol. A universal complementary DNA synthesis kit (Invitrogen; Thermo Fisher Scientific, Inc.) was utilized for RT. Each reaction mixture contained $0.5 \mu 1$ random primers $(0.2 \mu \mathrm{g} / \mu \mathrm{l})$ and $1 \mu \mathrm{l}$ SuperScript III reverse transcriptase (200 U/ $/ \mu 1)$. The specific primers used are listed in Table I. PCR was performed using the SYBR qPCR mix kit. The PCR conditions were as follows: Initial denaturation at $95^{\circ} \mathrm{C}$ for $2 \mathrm{~min}$, followed by 40 cycles of denaturation at $95^{\circ} \mathrm{C}$ for $10 \mathrm{sec}$, annealing at $60^{\circ} \mathrm{C}$ for $30 \mathrm{sec}$ and elongation at $70^{\circ} \mathrm{C}$ for $45 \mathrm{sec}$. PCR was performed using a CFX96 Touch $^{\mathrm{TM}}$ Real-Time PCR Detection system (Bio-Rad Laboratories, Inc., Hercules, CA, USA). Gene expression was determined and normalized to $\beta$-actin. The primers for rat $\beta$-actin were forward, 5'-AGGGAAATCGTGCGTGAC-3' and reverse,5'-CGCTCATTGCCGATAGTG-3'. The $2^{-\Delta \Delta C q}$ method was utilized to determine the relative gene expression (23). 
3'-UTR luciferase reporter assay. The transfection efficiency for delivering miRNA mimics to H9c2 cardiomyocytes was low when the luciferase reporter assay was first performed, potentially as a result of co-transfection of miRNA with luciferase vectors. Therefore, luciferase reporter assays were then performed with 293 cells (CRL-1573, American Type Culture Collection, Manassas, VA, USA) according to protocols of previous studies (24-26). Luciferase reporter vectors driven by the respective putative miR-21 binding sequence in the 3'-UTR of A-kinase anchoring protein 8 (Akap8; CL853-Gluc-Cluc-AKAP8-3'UTR) and BRCA1 associated RING domain 1 (Bard1; CL852-Gluc-Cluc-BARD1-3'UTR) were constructed. The 293 cells were divided into 7 experimental groups $\left(5 \times 10^{5}\right.$ cells/well): i) Control (untreated); ii) Akap8-3'UTR+miR-21 negative control (NC); iii) Akap8-3'UTR+miR-21 mimics; iv) Akap8-3'UTR+miR-21 inhibitor; v) Bard1-3'UTR+miR-NC; vi) Bard1-3'UTR+miR-21 mimics; and vii) Bard1-3'UTR+miR-21 inhibitor. miR-21 mimics or miR-21 inhibitor and luciferase vector driven by Bard1 3'-UTR or luciferase vector driven by Akap8 3'-UTR were co-transfected into 293 cells according to the group design using Lipofectamine 2000 reagent. Luciferase activity in the culture supernatant was measured using a Dual-Luciferase Reporter Assay system (cat. no. E1910; Promega Corp., Madison, WI, USA) at $36 \mathrm{~h}$ after transfection following manufacturer's protocols. The firefly luciferase activity, expressed relative light units was normalized to Renilla luciferase activity for each sample. Samples were analyzed using TargetScan (www.targetscan.org). Experiments were performed in triplicate.

Statistical analysis. Statistical analysis was performed using GraphPad Prism version 5.0 (GraphPad Software Inc., La Jolla, CA, USA). The results are expressed as the mean \pm standard error of mean. Differences between 2 groups were assessed using Student's t-test. Differences among $\geq 3$ groups were compared by one-way analysis of variance followed by the Bonferroni post-hoc test. $\mathrm{P}<0.05$ was considered to indicate a statistically significant difference.

\section{Results}

Loperamide pre-treatment at 50 and $100 \mathrm{~nm}$ increases the viability of rat cardiomyocytes after hypoxia/reoxygenation. Compared with that in the control group, the viability of $\mathrm{H} 9 \mathrm{c} 2$ cells was significantly decreased after hypoxia/reoxygenation treatment $(\mathrm{P}<0.001$; Fig. 1). However, the viability of $\mathrm{H} 9 \mathrm{c} 2$ cells treated with 50 or $100 \mathrm{~nm}$ loperamide prior to hypoxia/reoxygenation was markedly increased compared with that in the H/R group. Cells treated with $50 \mathrm{~nm}$ loperamide had the highest viability. Therefore, $50 \mathrm{~nm}$ loperamide was used in the subsequent experiments.

The protective effect of loperamide on rat cardiomyocytes against hypoxia/reoxygenation-induced apoptosis is markedly decreased by miR-21 inhibitor and enhanced by miR-21 mimics. The transfection efficiency of miR-21 mimics and inhibitor was good for these experiments. The apoptotic cells were determined by quantifying the early

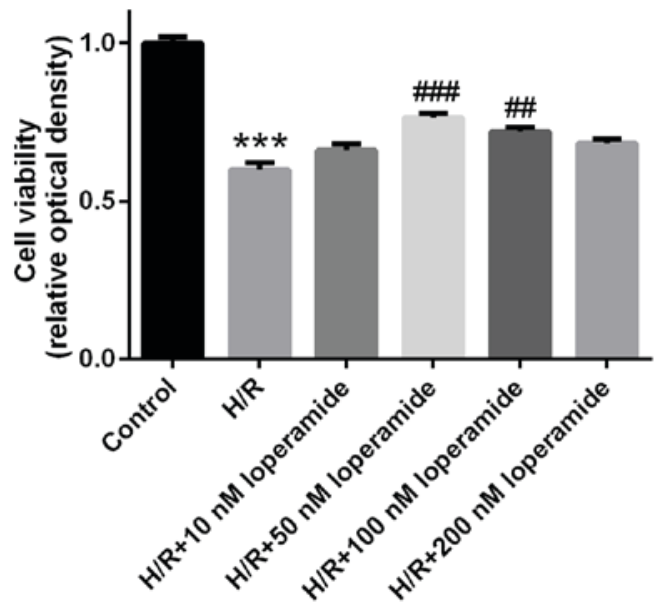

Figure 1. Pre-treatment with loperamide (50 and $100 \mathrm{~nm})$ increases the viability of rat cardiomyocytes after $\mathrm{H} / \mathrm{R}$. H9c2 rat cardiomyocytes were pre-treated with $0,10,50,100$ or $200 \mathrm{mM}$ loperamide for $24 \mathrm{~h}$ prior to hypoxia/reoxygenation treatment. The viability of $\mathrm{H} 9 \mathrm{c} 2$ cells was measured with a cell counting kit- 8 cell viability assay. After $H / R$ treatment, the viability of $\mathrm{H} 9 \mathrm{c} 2$ cells was significantly decreased, which was significantly inhibited pre-treatment with 50 or $100 \mathrm{~nm}$ loperamide. Cells treated with $50 \mathrm{~nm}$ loperamide had the highest viability. Therefore, $50 \mathrm{~nm}$ loperamide was used in subsequent experiments. Values are expressed as the mean \pm standard error of the mean ( $\mathrm{n}=3$ /group). ${ }^{* * *} \mathrm{P}<0.001$ vs. control group; ${ }^{\# \#} \mathrm{P}<0.01$,

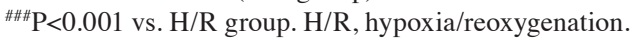

(quadrant 3) and late (quadrant 2) apoptotic cells. The apoptotic rate of rat cardiomyocytes was significantly increased after hypoxia/reoxygenation treatment as compared with that in the control group $(\mathrm{P}<0.001$; Fig. 2). Loperamide pre-treatment significantly protected $\mathrm{H} 9 \mathrm{c} 2$ cells against apoptosis after hypoxia/reoxygenation $(\mathrm{P}<0.001)$. The protective effect of loperamide was markedly decreased by miR-21 inhibitor $(\mathrm{P}<0.001)$ and enhanced by miR-21 mimics (P<0.001; Fig. 2).

The protective effect of loperamide on rat cardiomyocytes against hypoxia/reoxygenation-induced reactive oxygen species production is markedly decreased by miR-21 inhibitor and enhanced by miR-21 mimics. The reactive oxygen species levels in rat cardiomyocytes increased significantly after hypoxia/reoxygenation treatment as compared with those in the control group $(\mathrm{P}<0.001$; Fig. 3). Compared with those in the H/R group, pre-treatment with loperamide markedly decreased the reactive oxygen species levels in $\mathrm{H} 9 \mathrm{c} 2$ cells after hypoxia/reoxygenation $(\mathrm{P}<0.001)$. The decrease was markedly attenuated by miR-21 inhibitor $(\mathrm{P}<0.001)$ and enhanced by miR-21 mimics $(\mathrm{P}<0.01$; Fig. 3$)$.

miR-21 regulates the expression of Akap8 and Bard1, and enhances the inhibitory effects of loperamide on Akap8 and Bardl expression in rat cardiomyocytes after hypoxia/reoxygenation. Among the deregulated genes identified using the mRNA chip assay, those associated with cardiomyocyte apoptosis were screened out by pathway enrichment analysis: Akap8, BRCA1 interacting protein C-terminal helicase 1 (Brip1), heat shock protein family A (Hsp70) member 14 (Hspa14), RAD51 paralog B (Rad 51b); MAX dimerization protein 4 , methylenetetrahydrofolate 


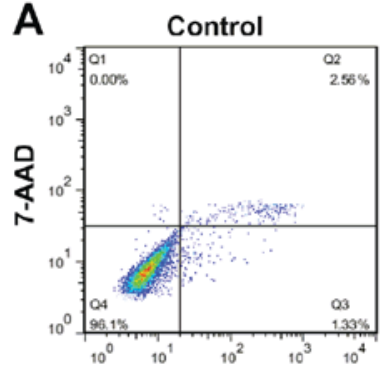

Annexin V-PE

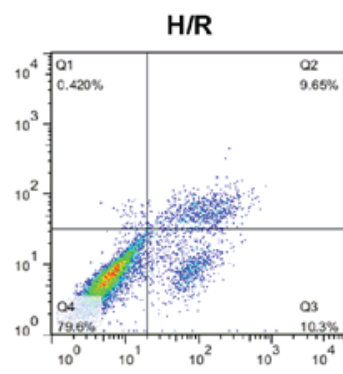

Annexin V-PE

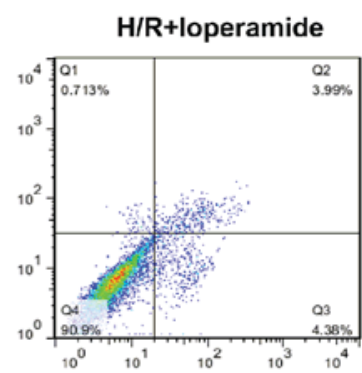

Annexin V-PE

$H / R+$ loperamide+miR-21 inhibitor $\quad H / R+$ loperamide+miRNA-21 mimic
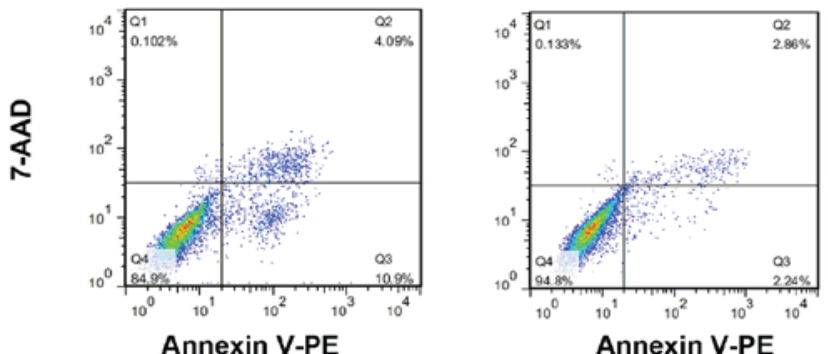

Annexin V-PE

B

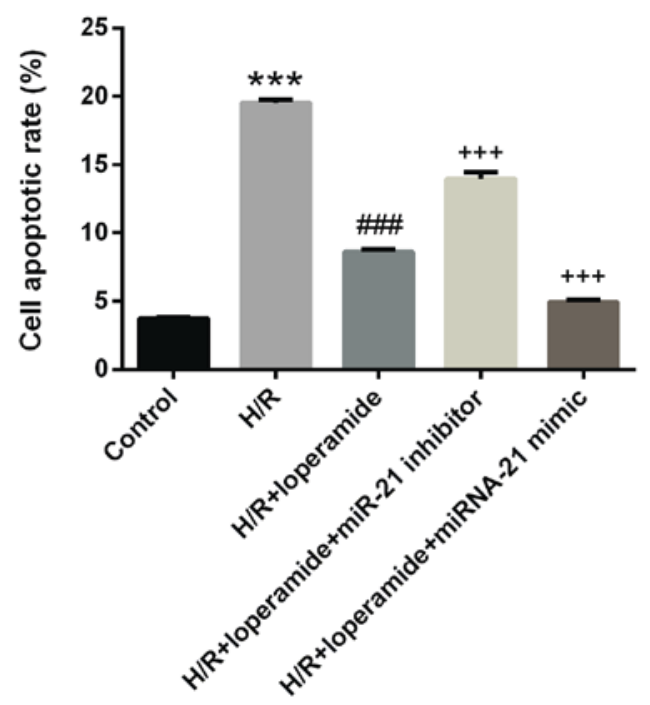

Figure 2. (A) Representative plot images of flow cytometry. (B) Statistical analysis of cell apoptotic rate. miR-21 inhibitor decreases and miR-21 mimics enhance the protective effect of loperamide against H/R-induced apoptosis of H9c2 rat cardiomyocytes. Cell apoptosis was detected with an Annexin V-PE/7-AAD apoptosis assay kit and flow cytometric analysis. The apoptotic rate was obtained by quantification of early (Q3) and late (Q2) apoptotic cells. The apoptotic rate of rat cardiomyocytes was significantly increased after hypoxia/reoxygenation treatment, as compared with that in the control group. Pre-treatment with loperamide significantly protected $\mathrm{H} 9 \mathrm{c} 2$ cells against apoptosis after $\mathrm{H} / \mathrm{R}$. The protective effect was markedly decreased by miR-21 inhibitor and enhanced by miR-21 mimics. Values are expressed as the mean \pm standard error of the mean ( $\mathrm{n}=3 /$ group) ${ }^{* * *} \mathrm{P}<0.001$ vs. control group; ${ }^{\# \# t} \mathrm{P}<0.001$ vs. $\mathrm{H} / \mathrm{R}$ group; ${ }^{+++} \mathrm{P}<0.001$ vs. $\mathrm{H} / \mathrm{R}+$ loperamide group. $\mathrm{H} / \mathrm{R}$, hypoxia/reoxygenation; miR, microRNA; PE, phycoerythrin; 7-AAD, 7-aminoactinomycin $\mathrm{D}$; $\mathrm{Q} 2$, quadrant 2.

dehydrogenase, cyclohydrolase and formyltetrahydrofolate synthetase and Bard1. The results of the mRNA chip assay were confirmed by RT-qPCR. The relative expression of Akap8 and Bard1 was consistent with the experimental results on apoptosis and reactive oxygen species in the 5 groups (Fig. 4). The expression of Brip1, Akap8, Rad51b, Hspa14 and Bard1 in rat cardiomyocytes increased significantly after hypoxia/reoxygenation treatment as compared with that in the control group $(\mathrm{P}<0.001$; Fig. 4). Compared with that in the H/R group, pre-treatment with loperamide markedly decreased the expression of Brip1, Akap8 and Bard1 in H9c2 cells after hypoxia/reoxygenation $(\mathrm{P}<0.001)$. The decrease in the expression of Akap8 and Bard1 was markedly attenuated by miR-21 inhibitor $(\mathrm{P}<0.001$ for Akap8; $\mathrm{P}<0.01$ for Bard1) and enhanced by miR-21 mimics $(\mathrm{P}<0.001$; Fig. 4).

miR-21 directly targets the 3'-UTR of Akap8 and Bardl $m R N A$. The direct binding of miR-21 to its putative binding sequences in the 3'-UTR of Akap8 and Bard1 was demonstrated using a Dual-Luciferase Reporter Assay system. At $36 \mathrm{~h}$ after co-transfection with reporter vectors driven by the binding regions in the 3'-UTR of Akap8 or Bard1 and miR-21 mimics or inhibitor, the luciferase activity in the different groups was determined. miR-21 mimics decreased the expression of Akap8 $(\mathrm{P}<0.001)$ and Bard1 $(\mathrm{P}<0.01)$, whereas miR-21 inhibitor increased the expression 

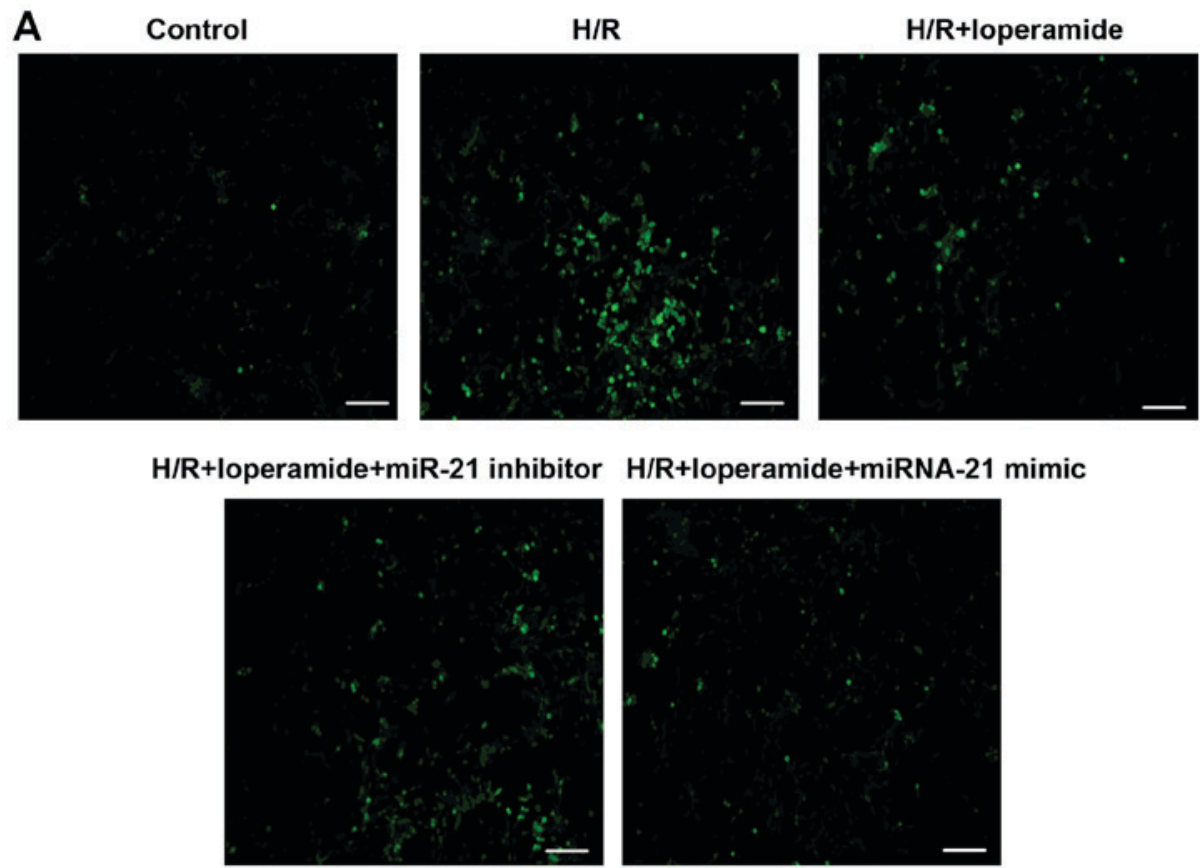

B

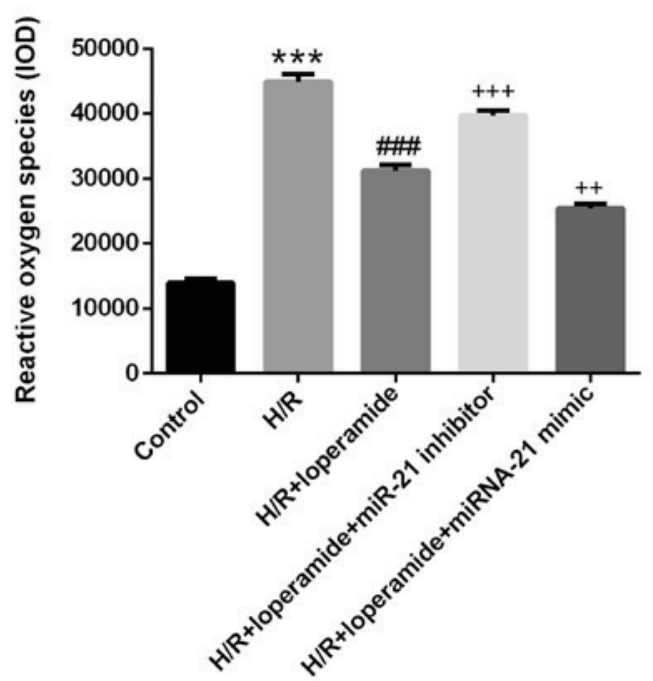

Figure 3. miR-21 inhibitor decreases and miR-21 mimics enhance the protective effect of loperamide against H/R-induced reactive oxygen species production in H9c2 rat cardiomyocytes. (A) Representative images for the detection of reactive oxygen species (scale bar, $100 \mu \mathrm{m}$ ). (B) Quantified levels of reactive oxygen species in the different experimental groups. Reactive oxygen species were detected with a reactive oxygen species assay kit. The reactive oxygen species levels in rat cardiomyocytes were significantly increased after $\mathrm{H} / \mathrm{R}$ treatment, as compared with those in the control group. Compared with the group treated by $\mathrm{H} / \mathrm{R}$ alone, pre-treatment with loperamide markedly decreased reactive oxygen species in H9c2 cells after H/R. The decrease was markedly alleviated by miR-21 inhibitor and enhanced by miR-21 mimics. Values are expressed as the mean \pm standard error of the mean $(\mathrm{n}=3 / \mathrm{group})$. ${ }^{* * * *} \mathrm{P}<0.001 \mathrm{vs}$. control group;

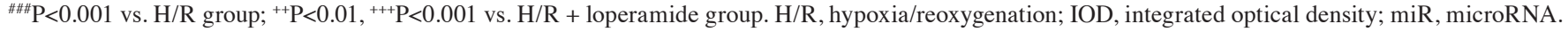

of Adap8 and Bard1 (P<0.01; Fig. 5). The putative miR-21 binding sites in the 3'-UTR of Akap8 and Bard1 mRNA are presented in Fig. 6.

\section{Discussion}

In the present study, it was demonstrated that the protective effect of loperamide on rat cardiomyocytes against hypoxia/reoxygenation-induced apoptosis and reactive oxygen species production was markedly enhanced by miR- 21 . miR-21 directly targets the 3'-UTR of Akap8 and Bard1 mRNA, and enhances the inhibitory effects of loperamide on the expression of Akap8 and Bard1 in rat cardiomyocytes after hypoxia/reoxygenation.
Ischemia/reperfusion injury or hypoxia/reoxygenation injury is the tissue and cell damage caused when blood supply and/or oxygen returns to tissue and cells after a period of ischemia or lack of oxygen. The restoration of the circulation and oxygen supply results in inflammation and oxidative damage via the induction of oxidative stress rather than restoration of normal function. Activated endothelial cells and other cell types produce more reactive oxygen species following reperfusion and reoxygenation, and the imbalance results in a subsequent inflammatory response (27). The restored blood flow and oxygen within cells and their associated free radicals damage cellular proteins, DNA and the plasma membrane. Damage to the cell membrane may in turn cause the release of more free radicals (28). In addition, ischemic tissue and hypoxic cells have 

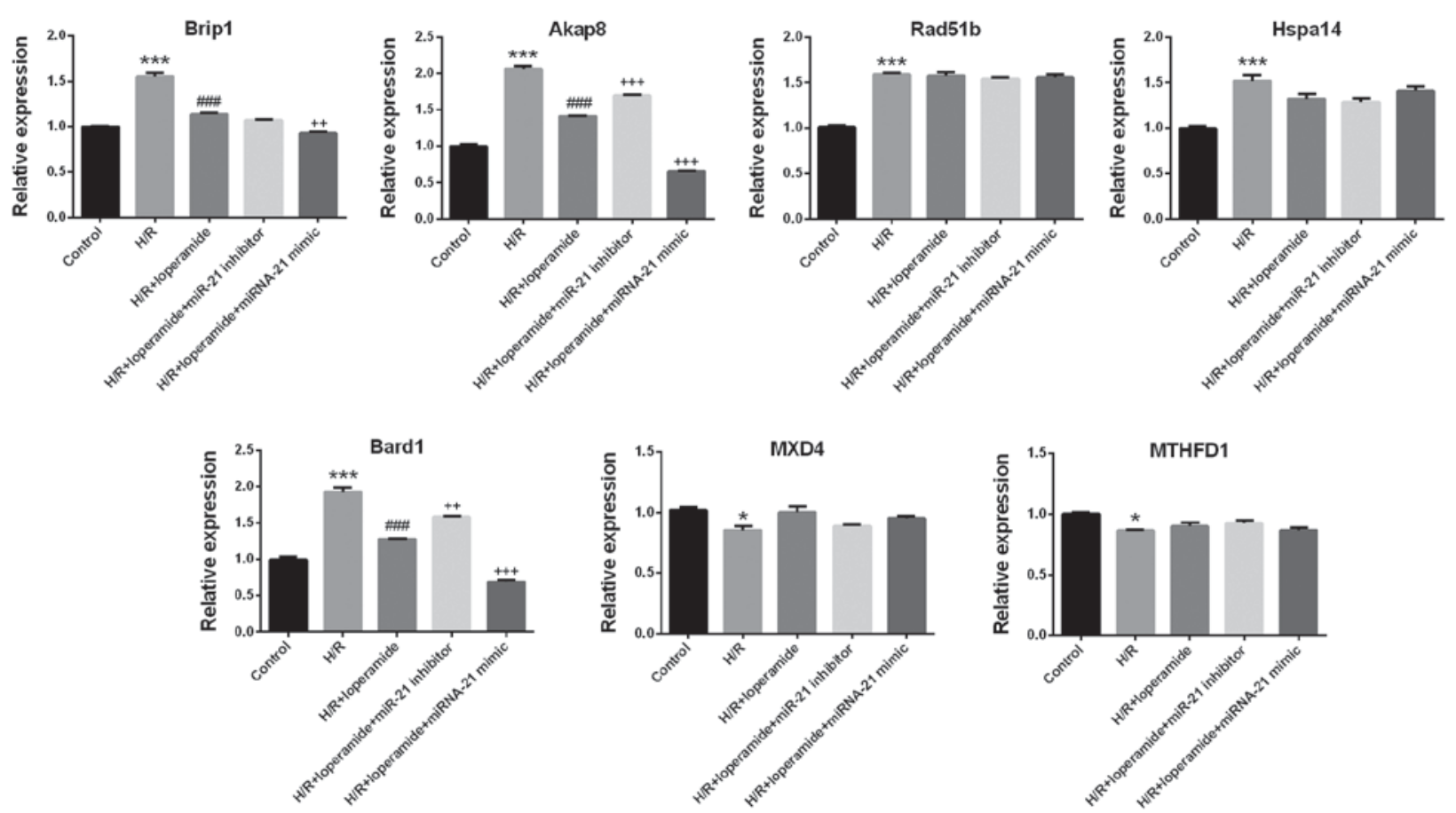

Figure 4. Akap8 and Bard1 expression is regulated by miR-21, and the inhibitory effects of loperamide on Akap8 and Bard1 expression in H9c2 rat cardiomyocytes after H/R are enhanced by miR-21. Following loperamide pre-treatment and H/R, miR-21-regulated genes were screened by mRNA chip. A pathway enrichment analysis was applied to screen out genes associated with cardiomyocyte apoptosis: Brip1, Akap8, Rad51b, Hspa14, Bard1, MXD4 and MTHFD1. The results of the mRNA chip analysis were confirmed by reverse transcription quantitative polymerase chain reaction. The relative expression of Akap8 and Bard1 was consistent with the results on apoptosis and reactive oxygen species in the experimental groups. The expression of Brip1, Akap8, Rad51b, Hspa14 and Bard1 in rat cardiomyocytes was significantly increased after H/R treatment, as compared with that in the control group. Compared with the group treated with $\mathrm{H} / \mathrm{R}$ alone, pre-treatment with loperamide markedly decreased the expression of Brip1, Akap8 and Bard1 in H9c2 cells after H/R. The decrease in expression of Akap8 and Bard1 was markedly alleviated by miR-21 inhibitor and enhanced by miR-21 mimics. Values are expressed as the mean \pm standard error of the mean ( $\mathrm{n}=3 /$ group). ${ }^{*} \mathrm{P}<0.05,{ }^{* * * *} \mathrm{P}<0.001$ vs. control group; ${ }^{\# \#+} \mathrm{P}<0.001 \mathrm{vs} . \mathrm{H} / \mathrm{R}$ group; ${ }^{++} \mathrm{P}<0.01,{ }^{+++} \mathrm{P}<0.001 \mathrm{vs}$. $\mathrm{H} / \mathrm{R}+\mathrm{loperamide}$ group. H/R, hypoxia/reoxygenation; Akap8, A-kinase anchoring protein 8; Bard1, BRCA1-associated RING domain 1; Brip1, BRCA1 interacting protein C-terminal helicase 1; Hspa14, heat shock protein family A (Hsp70) member 14; Rad 51b, RAD51 paralog B; MXD4, MAX dimerization protein 4; MTHFD1, methylenetetrahydrofolate dehydrogenase, cyclohydrolase and formyltetrahydrofolate synthetase 1; miR, microRNA.

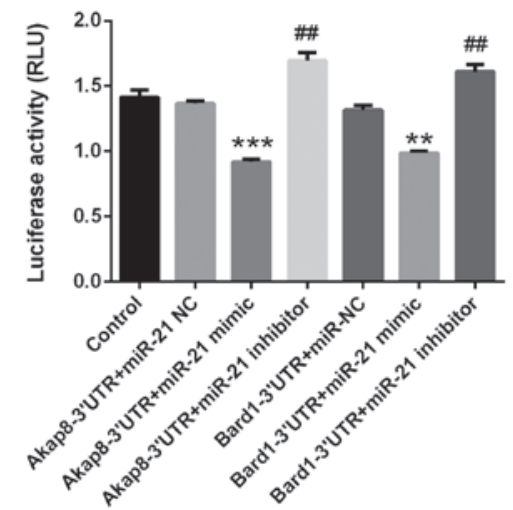

Figure 5. miR-21 directly targets the 3'-UTR of Akap8 and Bard1 mRNA. Luciferase reporter vectors containing the putative binding sequence in the 3'-UTR of Akap8 (CL853-Gluc-Cluc-AKAP8-3'UTR) or Bard1 (CL852-Gluc-Cluc-BARD1-3'UTR) were constructed. 293 cells were divided into 7 experimental groups. miR-21 mimics or miR-21 inhibitor or the respective controls were co-transfected with reporter vector containing the Akap8 or the Bard1 3'-UTR into 293 cells according to using Lipofectamine ${ }^{\circledR}$ 2000 reagent. At $36 \mathrm{~h}$ after transfection, the luciferase activity in the culture supernatant was measured using the Dual-Luciferase Reporter Assay system. miR-21 mimics decreased and miR-21 inhibitor increased the luciferase activity of the reporter vectors driven by the Adap8 and Bard1 3'-UTRs. Values are expressed as the mean \pm standard error of the mean ( $n=3$ /group). ${ }^{* *} \mathrm{P}<0.01,{ }^{* * *} \mathrm{P}<0.001$, mimics vs. $\mathrm{NC}$ and control group; ${ }^{\# *} \mathrm{P}<0.001$, inhibitor vs. NC and control group. NC, negative control; RLU, relative light units; Akap8, A-kinase anchoring protein 8; Bard1, BRCA1-associated RING domain 1; UTR, untranslated region. a decreased reactive oxygen species scavenger function due cell injury (29). Such reactive oxygen species may also act in redox signaling to turn on apoptosis.

The present study revealed that loperamide protected rat cardiomyocytes against hypoxia/reoxygenation-induced apoptosis and reactive oxygen species production. Morphine, another $\mu$-receptor agonist, was reported to protect against acute myocardial ischemia/reperfusion injury in rats $(30,31)$. Morphine post-conditioning protected against reperfusion injury via inhibiting c-Jun N-terminal kinase/p38 mitogen-activated protein kinase (MAPK) and mitochondrial permeability transition pore signaling pathways (32). Furthermore, morphine administration at reperfusion failed to improve post-ischemic cardiac function but limited myocardial injury via phosphorylation of HSP27 (33). Morphine pre-conditioning in the delayed phase was reported to exert protective effects on myocardial ischemia/reperfusion injury by inhibiting myocardial p38 MAPK activity and decreasing tumor necrosis factor- $\alpha$ production in rabbits (34). Evidence for the effects of loperamide on hypoxia/reoxygenation injury of cardiomyocytes is scarce. To the best of our knowledge, the present study was the first to demonstrate the protective role of loperamide against hypoxia/reoxygenation-induced apoptosis and reactive oxygen species production in rat cardiomyocytes.

In addition, it was demonstrated that the protection of rat cardiomyocytes against hypoxia/reoxygenation-induced injury 


\begin{tabular}{|l|c|c|c|c|c|c|c|}
\hline A & $\begin{array}{c}\text { Predicted consequential pairing of target } \\
\text { region (top) and miRNA (bottom) }\end{array}$ & $\begin{array}{c}\text { Site } \\
\text { type }\end{array}$ & $\begin{array}{c}\text { Context } \\
++ \\
\text { score }\end{array}$ & $\begin{array}{c}\text { Context++ } \\
\text { score } \\
\text { percentile }\end{array}$ & $\begin{array}{c}\text { Weighted } \\
\text { context++ } \\
\text { score }\end{array}$ & $\begin{array}{c}\text { Conserve } \\
\text { d branch } \\
\text { length }\end{array}$ & Pct \\
\hline $\begin{array}{c}\text { Position 50-56 } \\
\text { of AKAP8 } \\
\text { 3'UTR } \\
\text { rno-miR-21-5D }\end{array}$ & 5'UGUGUUCUUGUUUUAUAAGCUG & 7 'AGU & -0.38 & 98 & -0.32 & 0 & $<0.1$ \\
\hline
\end{tabular}

\begin{tabular}{|c|c|c|c|c|c|c|c|}
\hline B & $\begin{array}{c}\text { Predicted consequential pairing of target } \\
\text { region (top) and miRNA (bottom) }\end{array}$ & $\begin{array}{c}\text { Site } \\
\text { type }\end{array}$ & $\begin{array}{c}\text { Context } \\
++ \\
\text { score }\end{array}$ & $\begin{array}{c}\text { Context++ } \\
\text { score } \\
\text { percentile }\end{array}$ & $\begin{array}{c}\text { Weighted } \\
\text { context++ } \\
\text { score }\end{array}$ & $\begin{array}{c}\text { Conserved } \\
\text { branch } \\
\text { length }\end{array}$ & Pct \\
\hline $\begin{array}{c}\text { Position } \\
\text { 1053-1060 of } \\
\text { BRAD1 3'UTR } \\
\text { rno-miR-21-5p }\end{array}$ & $\begin{array}{c}\text { 5'UUCGUGCAUCUUUGC-AUAAGCUA } \\
\text { 3'AGUUGUAGUCAGACUAUUCGAU }\end{array}$ & $8 \mathrm{mer}$ & -0.11 & 79 & -0.05 & 0 & $<0.1$ \\
\hline
\end{tabular}

Figure 6. Predicted consequential pairing of miR-21 and 3'-UTR sequences of Akap8 and Bard1 mRNA. (A) Predicted consequential pairing of miR-21 and a seed sequence from the 3'-UTR of Akap8 mRNA; (B) Predicted consequential pairing of miR-21 and a seed sequence from the 3'-UTR of Bard1 mRNA. UTR, untranslated region; Akap8, A-kinase anchoring protein 8; Bard1, BRCA1-associated RING domain 1; miR, microRNA; rno, rattus norvegicus.

by loperamide was markedly enhanced by miR-21. It was also unveiled that miR-21 directly targeted the 3'-UTR of Akap8 and Bard1 mRNA, and enhanced the inhibitory effects of loperamide on Akap8 and Bard1 expression in rat cardiomyocytes after hypoxia/reoxygenation. miR-1 and -21 were reported to exert synergistic effects against hypoxia-induced cardiomyocyte apoptosis by activating AKT and blocking hypoxia-induced upregulation of p53 (35). miR-21 was revealed to attenuate hepatocyte hypoxia/reoxygenation injury by inhibiting the PTEN/PI3K/AKT signaling pathway (17). Rapamycin was reported to suppress hypoxia/reoxygenation-induced islet injury by upregulating miR-21 via the PI3K/AKT signaling pathway (18). Furthermore, the Akaps are a group of structurally diverse proteins that bind to the regulatory subunit of protein kinase A and confine it to discrete locations within cells (36). Akap8 is involved in the regulation of structural changes of chromatin through nuclear tyrosine phosphorylation. Akap8/Akap95 has been reported to mediate apoptosis, and to serve as a carrier to transport caspase 3 from the cytoplasm to the nucleus to induce morphological changes in the nucleus (37). Akap8 was also reported to have an important role in the modulation of head size and may contribute to the risk of autism (38). Furthermore, Bard1 is involved in apoptosis through binding and stabilizing p53 independently of BRCA1 (39). The expression of Bard1 is upregulated by genotoxic stress. Bard1 is also vital in the rapid relocation of BRCA1 to DNA damage sites (40). In the present study, it was revealed that pre-treatment with loperamide and transfection of miR-21 mimics decreased the expression of the pro-apoptotic genes Akap8 and Bard1 in rat cardiomyocytes after hypoxia/reoxygenation. miR-21 was demonstrated to directly target the 3'-UTR of Akap8 and Bard1 mRNA. Despite of minor limitation that we did not construct vectors containing a mutated 3 'UTR sequence, these results provide an explanation for the protective role of loperamide and miR-21 against hypoxia/reoxygenation-induced apoptosis and reactive oxygen species production in cardiomyocytes.

In conclusion, the present study demonstrated that the protection of rat cardiomyocytes against hypoxia/reoxygenation-induced apoptosis and reactive oxygen species production by loperamide is markedly enhanced by miR-21. miR-21 directly targets the 3'-UTR of Akap8 and Bard1 mRNA, and enhances the inhibitory effects of loperamide on Akap8 and Bard1 expression in rat cardiomyocytes after hypoxia/reoxygenation.

\section{Acknowledgements}

Not applicable.

\section{Funding}

The present study was supported by the Key Program of Health and Family Planning Commission of Shanghai, China (grant no. 201440024) and Key Project of Shanghai Municipal Health Bureau (grant no. 2016ZB0202).

\section{Availability of data and materials}

The analyzed data sets generated during the study are available from the corresponding author on reasonable request.

\section{Authors' contributions}

HS designed experiments and drafted manuscript.ZY analyzed and interpreted data. WZ and YZ collected experimental data. CY interpreted the data, provided experimental support and critically revised manuscript. CT designed experiments and critically revised manuscript. The final version of the manuscript has been read and approved by all authors and each author believes that the manuscript represents honest work.

\section{Ethics approval and consent to participate}

Not applicable.

\section{Patient consent for publication}

Not applicable. 


\section{Competing interests}

The authors declare that they have no competing interests.

\section{References}

1. White HD and Chew DP: Acute myocardial infarction. Lancet 372: 570-584, 2008

2. O'Gara PT, Kushner FG, Ascheim DD, Casey DE Jr, Chung MK, de Lemos JA, Ettinger SM, Fang JC, Fesmire FM, Franklin BA, et al: 2013 ACCF/AHA guideline for the management of ST-elevation myocardial infarction: A report of the American College of Cardiology Foundation/American Heart Association Task Force on Practice Guidelines. Circulation 127: e362-e425, 2013

3. Fernández-Jiménez R, García-Prieto J, Sánchez-González J, Agüero J, López-Martín GJ, Galán-Arriola C, Molina-Iracheta A, Doohan R, Fuster V and Ibáñez B: Pathophysiology underlying the bimodal edema phenomenon after myocardial ischemia/reperfusion. J Am Coll Cardiol 66: 816-828, 2015.

4. Bolli R, Becker L, Gross G, Mentzer R Jr, Balshaw D and Lathrop DA; NHLBI Working Group on the Translation of Therapies for Protecting the Heart from Ischemia: Myocardial protection at a crossroads: The need for translation into clinical therapy. Circ Res 95: 125-134, 2004.

5. Cannon RO III: Mechanisms, management and future directions for reperfusion injury after acute myocardial infarction. Nat Clin Pract Cardiovasc Med 2: 88-94, 2005

6. Ibáñez B, Heusch G, Ovize M and Van de Werf F: Evolving therapies for myocardial ischemia/reperfusion injury. J Am Coll Cardiol 65: 1454-1471, 2015.

7. MacDonald R, Heiner J, Villarreal J and Strote J: Loperamide dependence and abuse. BMJ Case Rep 2015: bcr2015209705, 2015.

8. Baker DE: Loperamide: A pharmacological review. Rev Gastroenterol Disord 7 (Suppl 3): S11-S18, 2007.

9. Kalgutkar AS and Nguyen HT: Identification of an $\mathrm{N}$-methyl-4-phenylpyridinium-like metabolite of the antidiarrheal agent loperamide in human liver microsomes: Underlying reason(s) for the lack of neurotoxicity despite the bioactivation event. Drug Metab Dispos 32: 943-952, 2004.

10. Wang XH, Zeng JF, Lin C and Chen SB: Effects of morphine and sufentanil preconditioning against myocardial ischemic-reperfusion injury in rabbits. Int J Clin Exp Med 8 : 15692-15699, 2015.

11. Bartel DP: MicroRNAs: Genomics, biogenesis, mechanism, and function. Cell 116: 281-297, 2004.

12. Asangani IA, Rasheed SA, Nikolova DA, Leupold JH, Colburn NH, Post S and Allgayer H: MicroRNA-21 (miR-21) post-transcriptionally downregulates tumor suppressor Pdcd4 and stimulates invasion, intravasation and metastasis in colorectal cancer. Oncogene 27: 2128-2136, 2008.

13. Frankel LB, Christoffersen NR, Jacobsen A, Lindow M, Krogh A and Lund AH: Programmed cell death 4 (PDCD4) is an important functional target of the microRNA miR-21 in breast cance cells. J Biol Chem 283: 1026-1033, 2008.

14. Meng F, Henson R, Wehbe-Janek H, Ghoshal K, Jacob ST and Patel T: MicroRNA-21 regulates expression of the PTEN tumor suppressor gene in human hepatocellular cancer Gastroenterology 133: 647-658, 2007.

15. Si ML, Zhu S, Wu H, Lu Z, Wu F and Mo YY: miR-21-mediated tumor growth. Oncogene 26: 2799-2803, 2007.

16. Zhu S, Si ML, Wu H and Mo YY: MicroRNA-21 targets the tumor suppressor gene tropomyosin 1 (TPM1). J Biol Chem 282: 14328-14336, 2007.

17. Lu X, Sun C, Zheng D, Liu R, Wei X and Wu Z: [The miR-21 attenuates hepatocyte hypoxia/reoxygenation injury via inhibiting PTEN/PI3K/AKT signaling pathway]. Xi Bao Yu Fen Zi Mian Yi Xue Za Zhi 33: 497-502, 2017 (In Chinese).

18. Zhang Y, He S, Du X, Jiang Y, Tian B and Xu S: Rapamycin suppresses hypoxia/reoxygenation-induced islet injury by up-regulation of miR-21 via PI3K/Akt signalling pathway. Cell Prolif 50, 2017.

19. Tee YT, Han CP, Ko JL, Chen GD, Yang SF, Chen SC, Tsai HJ, Lin LY and Wang PH: Evaluation of matrix metalloproteinase 2 expression in cervical carcinogenesis using tissue array and integrated optical density for immunoreactivity. Reprod Sci 14: 719-726, 2007.
20. Kanehisa M, Goto S, Kawashima S, Okuno Y and Hattori M The KEGG resource for deciphering the genome. Nucleic Acids Res 32 (Database Issue): D277-D280, 2004.

21. Yi M, Horton JD, Cohen JC, Hobbs HH and Stephens RM: WholePathwayScope: A comprehensive pathway-based analysis tool for high-throughput data. BMC Bioinformatics 7: 30, 2006.

22. Draghici S, Khatri P, Tarca AL, Amin K, Done A, Voichita C, Georgescu C and Romero R: A systems biology approach for pathway level analysis. Genome Res 17: 1537-1545, 2007.

23. Livak KJ and Schmittgen TD: Analysis of relative gene expression data using real-time quantitative PCR and the 2(-Delta Delta $\mathrm{C}(\mathrm{T})$ ) method. Methods 25: 402-408, 2001

24. Xia M, Huang R, Guo V, Southall N, Cho MH, Inglese J, Austin CP and Nirenberg M: Identification of compounds that potentiate CREB signaling as possible enhancers of long-term memory. Proc Natl Acad Sci USA 106: 2412-2417, 2009.

25. Brunzell DH, Mineur YS, Neve RL and Picciotto MR: Nucleus accumbens CREB activity is necessary for nicotine conditioned place preference. Neuropsychopharmacology 34: 1993-2001, 2009.

26. Cali JJ, Niles A, Valley MP, O'Brien MA, Riss TL and Shultz J: Bioluminescent assays for ADMET. Expert Opin Drug Metab Toxicol 4: 103-120, 2008 .

27. Carden DL and Granger DN: Pathophysiology of ischaemia-reperfusion injury. J Pathol 190: 255-266, 2000.

28. Caraceni P, VAN Thiel DH and Borle AB: Time course of free radicals formation, lipid peroxidation, and reoxygenation injury in perfused rat hepatocytes. Ann N Y Acad Sci 723: 356-359, 1994.

29. Burn BR and Varner KJ: Environmentally persistent free radicals compromise left ventricular function during ischemia/reperfusion injury. Am J Physiol Heart Circ Physiol 308: H998-H1006, 2015.

30. Zhang ZY, Li PJ, Chen TD, Yang L, Zhao JH, Na T and Tang XQ: Protective effect and mechanism of morphine on acute myocardial ischemia/reperfusion injury in rats. Zhongguo Wei Zhong Bing Ji Jiu Yi Xue 16: 656-659, 2004 (In Chinese).

31. Chang WL, Lee SS and Su MJ: Attenuation of post-ischemia reperfusion injury by thaliporphine and morphine in rat hearts. J Biomed Sci 12: 611-619, 2005.

32. Chen Z, Zhang X, Liu Y and Liu Z: Morphine postconditioning protects against reperfusion injury via inhibiting JNK/p38 MAPK and mitochondrial permeability transition pores signaling pathways. Cell Physiol Biochem 39: 61-70, 2016.

33. Mourouzis I, Saranteas T, Perimenis P, Tesseromatis C, Kostopanagiotou G, Pantos C and Cokkinos DV: Morphine administration at reperfusion fails to improve postischaemic cardiac function but limits myocardial injury probably via heat-shock protein 27 phosphorylation. Eur J Anaesthesiol 26: 572-581, 2009.

34. Ran K, Gong ZX, Yang DL, Chang YT, Duan KM and Ou YW: Effect of morphine preconditioning in the delayed phase on the expression of p38 mitogen-activated protein kinase in a rabbit model of myocardial ischemia-reperfusion injury. Genet Mol Res 14: 6642-6648, 2015.

35. Xu Y, Zhu W, Wang Z, Yuan W, Sun Y, Liu H and Du Z: Combinatorial microRNAs suppress hypoxia-induced cardiomyocytes apoptosis. Cell Physiol Biochem 37: 921-932, 2015.

36. Eide T, Taskén KA, Carlson C, Williams G, Jahnsen T, Taskén K and Collas P: Protein kinase A-anchoring protein AKAP95 interacts with MCM2, a regulator of DNA replication. J Biol Chem 278: 26750-26756, 2003.

37. Kubota S, Morii M, Yuki R, Yamaguchi N, Yamaguchi $H$, Aoyama K, Kuga T, Tomonaga T and Yamaguchi N: Role for tyrosine phosphorylation of A-kinase anchoring protein 8 (AKAP8) in its dissociation from chromatin and the nuclear matrix. J Biol Chem 290: 10891-10904, 2015.

38. Nebel RA, Kirschen J, Cai J, Woo YJ, Cherian K and Abrahams BS: Reciprocal relationship between head size, an autism endophenotype, and gene dosage at $19 \mathrm{p} 13.12$ points to AKAP8 and AKAP8L. PLoS One 10: e0129270, 2015.

39. Irminger-Finger I, Leung WC, Li J, Dubois-Dauphin M, Harb J, Feki A, Jefford CE, Soriano JV, Jaconi M, Montesano R and Krause KH: Identification of BARD1 as mediator between proapoptotic stress and p53-dependent apoptosis. Mol Cell 8: 1255-1266, 2001

40. Li M and Yu X: Function of BRCA1 in the DNA damage response is mediated by ADP-ribosylation. Cancer Cell 23: 693-704, 2013.

This work is licensed under a Creative Commons Attribution-NonCommercial-NoDerivatives 4.0 International (CC BY-NC-ND 4.0) License. 